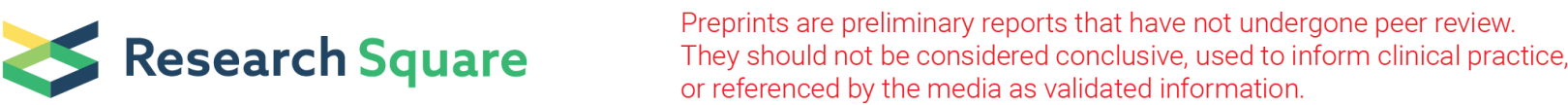

\section{An oral absorbent, AST-120, Restores Vascular Growth and Blood Flow in Ischemic Muscles in Diabetic Mice via Modulation of Macrophage Transition}

\author{
Hsin-Lei Huang \\ National Taipei University of Nursing and Health Sciences \\ Chin-Sung Kuo \\ Taipei Veterans General Hospital \\ Ting-Yung Chang \\ Taipei Veterans General Hospital \\ Ruey-Hsing Chou \\ Taipei Veterans General Hospital \\ I-Chun Chen \\ National Yang-Ming University \\ Fu-Chen Yang \\ National Yang-Ming University \\ Nien-Jung Chen \\ National Yang-Ming University \\ Der-Cherng Tarng \\ Taipei Veterans General Hospital \\ Shing-Jong Lin \\ Taipei Veterans General Hospital \\ Chih-Cheng Wu \\ National Taiwan University Hospital \\ Po-Hsun Huang ( $\nabla$ huangbs@vghtpe.gov.tw ) \\ Division of Cardiology, Taipei Veteran General Hospital
}

Original investigation

Keywords: diabetes, advanced glycation end products, AST-120, macrophage, neovascularization

Posted Date: May 28th, 2020

DOI: https://doi.org/10.21203/rs.3.rs-28913/v1 
License: (c) (i) This work is licensed under a Creative Commons Attribution 4.0 International License. Read Full License

Version of Record: A version of this preprint was published at Journal of Molecular and Cellular Cardiology on June 1st, 2021. See the published version at https://doi.org/10.1016/j.yjmcc.2021.03.001. 


\section{Abstract \\ Background}

Diabetes has a pronounced effect on the peripheral vasculature. The accumulation of advanced glycation end products (AGEs) is regarded as the crucial mechanism responsible for vascular damage in diabetes, but it is not easy to be avoided from food. In this study, we aimed to investigate the effects of an oral absorbent, AST-120, on the accumulation of AGEs and changes in blood flow recovery in diabetic mice.

\section{Methods}

The mice were divided into four groups, wild-type (WT) mice without treatment, WT mice treated with 5\% AST-120 mixed into with pulverized chow, streptozotocin-induced diabetes mellitus (DM) mice, and DM mice treated with 5\% AST-120. Six weeks after hind-limb ischemia surgery, blood flow reperfusion, histology, plasma AGE, and cytokine were examined. Bone marrow cells were cultured and derived into macrophages to evaluate the effects of AGEs on macrophage polarization.

\section{Results}

Plasma AGEs were significantly increased in diabetic mice. AST-120 could bind to AGEs and reduced their plasma concentrations. Histological analysis revealed fewer collateral vessels with corresponding impairment of blood flow recovery in diabetic mice. In these mice, AGE-positive and AGE receptor-positive macrophages were numerous in ischemic limbs when compared with non- diabetic mice. In diabetic mice, macrophages in ischemic tissues demonstrated greater M1 polarization than M2 polarization; this pattern was reversed in the AST-120 treatment group. The change in macrophage polarization was associated with corresponding expression of pro-inflammatory and pro-angiogenic cytokines in the ischemic tissues. In cell cultures, AGEs triggered the transformation of bone marrow-derived macrophages into M1 phenotype. The alterations in the polarization of macrophages were reversed after treatment with AST-120.

\section{Conclusions}

Oral administration of AST-120 decreased the serum levels of AGEs in diabetic mice and improved neovascularization of ischemic limbs. This benefit may be due to, at least partially, the alterations in macrophage polarization and the associated changes in inflammatory and angiogenic cytokines.

\section{Background}


Cardiovascular complications are the leading cause of morbidity and mortality in patients with diabetes mellitus. Diabetes has a pronounced effect on the peripheral vasculature, which results in high nontraumatic amputation rates. The management of peripheral arterial disease in patients with diabetes is challenging, and the outcomes of revascularization are poor [1]. Patients with diabetes have diminished capacity for neovascularization to overcome the reduction in blood flow following arterial stenosis or occlusion [2]. This probably contributes to the adverse outcomes when limb ischemia develops in these patients. Few studies have specifically evaluated the effects of diabetes on angiogenesis in peripheral vascular diseases. Furthermore, the mechanisms underlying the impaired neovascularization remain largely undefined, resulting in limited therapeutic strategies to improve critical limb ischemia.

The accumulation of advanced glycation end products (AGEs) is a crucial mechanism responsible for the vascular damage in diabetes [3-5]. AGEs are post-translational modifications of proteins in which amino acids and carbohydrates interact via an irreversible, non-enzymatic process. Chronic hyperglycemia and the altered redox state in diabetes result in increased formation of AGEs. One mechanism by which the effects of AGEs are elicited is through their binding with the receptor for advanced glycation end products (RAGE) [6-8]. Binding of AGEs with RAGE activates several intracellular pathways, resulting in the upregulation of multiple pro-inflammatory genes [9]. In diabetes, RAGE has been demonstrated to be elevated and co-localized with AGEs in a variety of tissues, especially in the affected vasculature [10]. Activation of RAGE has also been observed in endothelial cells, vascular smooth muscle cells, and macrophages [11], which play significant roles in neovascularization following tissue ischemia.

AST-120 (Kremezin $\left.{ }^{\circledR}\right)$ is an oral absorbent that can bind to many low-molecular-weight (100$10,000 \mathrm{kDa}$ ) compounds, including carboxymethyl lysine (CML), a well-characterized AGE [12]. AST-120 has been demonstrated to decrease the serum levels of AGEs in patients with chronic kidney disease (CKD) but without diabetes [12-14]. We hypothesized that AGEs and RAGE signaling could play inhibitory roles in angiogenesis by promoting excessive inflammation in ischemic limbs of patients with diabetes. To test this hypothesis, we investigated the effects of treatment with AST-120 on the accumulation of AGEs in diabetic mice and evaluated its impact on inflammation, neovascularization, and reperfusion in a hind-limb ischemia model.

\section{Methods}

\section{Food Preparation and AGE-AST120 Binding Assay}

Regular pulverized chow food was prepared without heat exposure (Regular-AGE); it contained 18\% protein, $58 \%$ carbohydrate, $7.5 \%$ fat, and $3.73 \mathrm{kcal} / \mathrm{g}$. Cooked pulverized chow food was prepared by heating at $125^{\circ} \mathrm{C}$ for $60 \mathrm{~min}$. Based on the measurement by an AGE-sensitive enzyme-linked immunosorbent assay (ELISA), this preparation contained $200 \mathrm{ng} / \mathrm{ml}$ of AGEs (Fig. 1B) and was used as a high-AGE diet. The concentration of CML demonstrated a similar pattern of two-fold difference between regular chow food and cooked chow food (Fig. 1C). To identify the binding effect of AST-120 in regular chow food or cooked chow food, both foods were individually mixed with 5\% AST-120 (Kremezin, Kureha 
Corporation, Osaka, Japan) in water. After 16 hours of incubation at $37^{\circ} \mathrm{C}$, the supernatant was measured using AGE and CML ELISA kits.

\section{Animals}

Eight-week-old male wild-type (WT) FVB mice were used in this study. The mice were divided into four groups based on the food they received (Fig. 1A): regular pulverized chow food (Chow food), 5\% AST-120 mixed with regular chow food (Chow food + AST-120), cooked pulverized chow food (Cooked chow food), and 5\% AST-120 mixed with cooked chow food (Cooked chow food + AST-120). After six weeks of the food consumption, the mice were euthanized., and their plasma samples were obtained for AGEs and CML measurements. In this experiment, intraperitoneal injection of tribromoethanol (avertin; 225-240 mg / kg body weight) was used as the anesthetic agent. After confirming the mouse in anesthesia, it was sacrificed by bleeding.

The mice were rendered diabetic by intraperitoneal injection of saline or streptozotocin (STZ: 5 days, 50 mg/kg in citrate buffer, 100 mmol/L, pH 4.5; Sigma, St. Louis, MO, USA). Blood glucose was measured 7 days after the first STZ injection using a blood glucose meter. Animals with blood glucose levels > $250 \mathrm{mg} / \mathrm{dL}$ were considered to be diabetic and were included for further experiments. These mice were divided into four groups: WT mice with no treatment (WT), WT mice treated with 5\% AST-120 mixed with pulverized chow (WT + AST-120), mice with STZ-induced diabetes mellitus (DM), and mice with STZinduced diabetes mellitus treated with 5\% AST-120 (DM + AST-120) (Fig. 1F). To confirm that the STZinduced DM mice represented a model of human type 2 diabetes, we performed blood glucose tests and intraperitoneal glucose tolerance tests (IPGTTs).

\section{AGE and CML Determination}

Circulating levels of AGEs and CML were determined using competitive ELISA kits.

\section{Hind-limb Ischemia Model}

Hind-limb ischemia was induced by excising the right femoral artery, as described previously [15]. The animals were anesthetized with intraperitoneal ketamine $(100 \mathrm{mg} / \mathrm{kg})$ and xylazine $(10 \mathrm{mg} / \mathrm{kg})$. The proximal and distal portions of the right femoral artery and the distal portion of the right saphenous artery were ligated. Subsequently, the arteries and all the branches were dissected free and excised.

\section{AGEs and Cytokine Assays}

Before hind-limb ischemia surgery, blood samples were collected from the facial veins of the mice. Plasma concentrations of AGEs were determined using a commercial enzyme-linked immunosorbent assay (Cell Biolabs, San Diego, CA, USA) according to the manufacturer's instructions. Two weeks after the hind-limb ischemia surgery, the concentrations of plasma cytokines and muscle extracted proteins, including tumor necrosis factor (TNF)-a, interleukin (IL)-6, chemokine (C-X-C motif) ligand 2 (Cxcl2), monocyte chemoattractant protein (MCP)-1, plasma macrophage colony-stimulating factor (M-CSF), vascular endothelial growth factor (VEGF), IL-4, and IL-10 were determined using the commercially 
available Procarta multiplex immunoassay kit (eBioscience, San Diego, CA, USA) according to the manufacturer's instructions.

\section{Laser Doppler Imaging}

The ratio of blood flow in the ischemic (right) limb to that in the non-ischemic (left) limb was measured with a laser Doppler perfusion imaging system (Moor Instruments Limited, Devon, UK). The mice were monitored using serial scanning of surface blood flow in the hind-limb before and after the hind-limb ischemia surgery, which was repeated weekly for four weeks.

\section{Histological Analysis}

Two weeks after the hind-limb ischemia surgery, the ischemic muscle tissue was collected. Tissue sections of $4 \mu \mathrm{m}$ thickness were cut for immunohistochemical staining. Capillaries were stained with rat monoclonal antibodies against CD31 (1:100 dilution; BD PharMingen, CA, USA). To assess angiogenesis, the smooth muscle cells were stained with $a$-smooth-muscle actin (SMA) antibody (1:250 dilution; Dako Cytomation, Denmark). AGEs accumulation was detected using a CML antibody (1:250 dilution; Abcam, Cambridge, MA, USA). Macrophage infiltration (total number of macrophages per region of interest [ROI]) was detected using rat polyclonal F4/80 antibody (1:50 dilution; Abcam). Thereafter, the tissue sections were incubated with an avidin-biotin-labeled polymer (Envision + Dual Link System-HRP; Dako). The numbers of CD31, a-SMA, and F4/80 immunopositive cells were counted in 10 randomly selected highpower fields at 400 - magnification using a multipurpose color image processor.

\section{RAGE Ligand Distribution, Macrophage Infiltration, and Characterization}

Immunohistochemistry was performed using goat anti-human RAGE (1:200 dilution; Genetex, CA, USA). Macrophages with the M1 phenotype were detected using rabbit inducible nitric oxide synthase (iNOS) antibody (1:500 dilution), and those with the M2 phenotype were detected using rabbit Arg1 antibody (1:100 dilution) in combination with the mouse macrophage marker F4/80 (1:100 dilution; all products from Abcam). Fluorescently conjugated secondary antibodies were used for signal detection. The M1 and M2 macrophages were counted in five randomly selected fields of each muscle section. Photographs of the stained histological sections were taken using a confocal microscope (Olympus Micro, Japan) and processed with the FV10-ASW 4.0 imaging system.

\section{Macrophage Cell Cultures}

To study the effects of AGEs on macrophage polarization, bone marrow cells were cultured and differentiated into macrophages of different phenotypes (Supplementary Fig. 1). Bone marrow cells were extracted from 6-week-old WT mice and cultured with M-CSF containing Royal Park Memorial Institute (RPMI) medium every other day. Well-differentiated bone marrow-derived macrophages (BMDMs) were harvested on day 5. BMDMs were incubated for 48 hours with different media: control medium, AGE $(100 \mu \mathrm{g} / \mathrm{ml})$, AST-120-pretreated AGE, high glucose (25 mmol/L D-glucose), high glucose + AGE, and high glucose + AST-120-pretreated AGE. Total RNA was isolated, and quantitative real-time polymerase chain 
reaction (PCR) was performed. The primers of IL-1b, iNOS, YM-1, Arginase-1 (Supplementary Fig. 2), and the mixture were purchased from SYBR Green systems (Roche LC480). BMDMs were triggered into M1 and M2 macrophages; IL-1b and iNOS are highly expressed in M1 macrophages, while arginase-1 and YM-1 are highly expressed in M2 macrophages. Data were calculated using the comparative Ct method $(\triangle \triangle \mathrm{Ct})$ and expressed as the -fold increase over the indicated controls.

\section{Statistical Analysis}

Data are presented as mean \pm standard error of mean. Statistical analysis was performed using the Student's unpaired t-test or one-way analysis of variance (ANOVA), followed by post-hoc Duncan's multiple-comparison test. Analyses were performed using SPSS version 16.0 (SPSS Inc., Chicago, IL, USA). $P$-values $<0.05$ were considered statistically significant.

\section{Results}

\section{AST-120 Reduces Plasma AGEs via Inhibition of Absorption of Dietary AGEs}

The content of AGEs in cooked chow is higher than that in the regular chow. AST-120 decreased the content of AGEs in both regular and cooked chows (Fig. 1B) as well as the content of CML (Fig. 1C). Therefore, AST-120 could adsorb AGEs and CML in the food. Plasma levels of AGEs and CML in mice that were fed cooked chow (high AGE content) were higher than those in mice that were fed regular chow (low AGE content). AST-120 reversed the elevated AGEs and CML levels in mice that were fed both regular chow and cooked chow (Fig. 1D, 1E). These observations confirm the role of dietary AGEs on the circulating AGE and CML levels and the effect of AST-120 therapy.

\section{AST-120 Reduced the Plasma AGE Concentration and Cytokine Levels in Diabetic Mice}

DM and DM + AST-120 mice had increased blood glucose levels under non-fasting conditions (Supplementary Fig. 3A) and on IPGTTs (Supplementary Fig. 3B) when compared with WT mice. Plasma AGE levels were elevated in DM mice when compared with those in WT mice; the AGE level was $67 \%$ lower in the DM + AST-120 group than that in the DM group (Fig. 1G). No differences in body weight were observed between the WT and WT + AST-120 groups (Supplementary Fig. 3C). Therefore, AST-120 reduced the plasma level of AGEs, but not the blood glucose level.

We assessed the expressions of circulating pro-inflammatory, anti-inflammatory, and angiogenic cytokines following the hind-limb ischemia surgery. Two weeks after the surgery, the plasma MCP-1 level was significantly higher in DM mice when compared with WT mice. In DM + AST-120 mice, a trend toward lower plasma MCP-1 level was observed when compared with DM mice (Fig. $1 \mathrm{H})$. On the other hand, the levels of IL-10 (Fig. 1I) and VEGF (Fig. 1J) were higher in WT mice when compared with DM mice. The DM + AST-120 group demonstrated a trend toward higher levels of plasma IL-10 and VEGF when 
compared with the DM group. There were no significant changes in the levels of other plasma cytokines, including TNF-a, IL-6, IL-4, and Cxcl2 (data not shown).

\section{AST-120 Treatment Reversed Blunted Perfusion Recovery and Impaired Neovascularization in the Ischemic Muscles of DM Mice}

Laser Doppler perfusion images demonstrated gradual reperfusion of the ischemic limbs with approximately $95 \%$ of the blood flow in the non-ischemic limbs at 4 weeks in WT mice (Fig. 2A). The blood flow recovery was $44 \%$ lower by 4 weeks in DM mice when compared with WT mice $(P<0.05)$ (Fig. 2B). In DM + AST-120 mice, the blood flow ratio in the ischemic limbs recovered significantly to the levels observed in the WT mice.

Two weeks following the hind-limb ischemia surgery, the densities of $\mathrm{CD} 31^{+}$capillaries (Fig. 2C, 2D) and

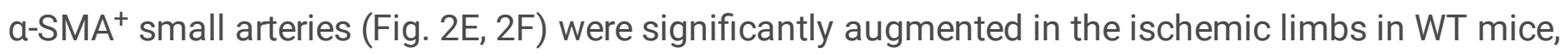
whereas no such increase was observed in DM mice. DM + AST-120 mice demonstrated significant recovery of $\mathrm{CD}_{3} 1^{+}$capillaries and $\mathrm{a}-\mathrm{SMA}^{+}$small arteries.

\section{Distribution of AGEs and RAGE in the Ischemic Limbs of DM Mice}

Our initial data suggested that neovascularization mechanisms were impaired in diabetes and restored, at least in part, by AST-120-mediated reduction of AGEs. To further examine this hypothesis, we quantified the number of CML- and RAGE-positive cells in ischemic muscles 2 weeks after the hind-limb ischemia injury (Fig. 3A-3D). Immunofluorescence staining revealed significantly higher numbers of CML-positive cells and RAGE-positive cells in DM mice than those in WT mice. Compared with DM mice, the number of CML-positive cells and AGE-positive cells in the ischemic limbs had significantly decreased after AST-120 treatment.

\section{Macrophage Infiltration in the Ischemic Limbs of DM Mice}

Macrophage infiltration between the myocytes was observed in ischemic tissues. DM mice demonstrated a significant increase in the total number of F4/80-positive macrophages when compared with WT mice. To clarify the impact of AGEs on the infiltration of macrophages, we assessed the co-localization of F4/80 and RAGE (Fig. 3E) using immunofluorescence staining and confocal microscopy images. The images demonstrated co-localization of F4/80 (yellow), RAGE (red), and the nuclear marker DAPI (4',6diamidino-2-phenylindole; blue) in the same cells infiltrated between the myocytes (Supplementary Fig. 4). DM mice demonstrated a significantly higher number of RAGE-positive macrophages in the ischemic muscles when compared with WT mice. After 5 weeks of AST-120 treatment, RAGE-positive macrophages had reduced significantly in DM + AST-120 mice when compared with DM mice (Fig. 3F). 


\section{Effects of Diabetes and AST-120 on M1 and M2 Macrophage Polarization}

We quantified the expression of M1 in comparison with M2 markers following the hind-limb ischemia surgery because these markers are generally associated with pro-inflammatory and pro-angiogenic signatures, respectively $[16,17]$. The ischemic limbs were stained for F4/80 and M1 (iNOS) and M2 (Arg1) markers. The data were analyzed by assessing the percentage of F4/80-positive macrophages (total macrophage number) that were double-stained with respective M1 or M2 markers under each experimental condition (Fig. 4A, 4B). The percentage of iNOS $^{+}$macrophages was significantly higher in DM mice than in WT mice (Fig. 4C), and it was significantly lower in DM + AST-120 mice than in DM mice (Fig. 4D). No difference was observed between WT and WT + AST-120 mice.

Analysis of M2 markers revealed a different pattern. The percentage of $\mathrm{Arg}^{+}{ }^{+}$macrophages was significantly lower in DM mice than that in WT mice. No difference was observed between WT and WT + AST-120 mice. However, DM + AST-120 mice demonstrated a trend toward a higher percentage of Arg $1^{+}$ macrophages when compared with DM mice (Fig. 4D). Collectively, the predominance of proinflammatory M1 macrophages was attenuated, as opposed to pro-angiogenic M2 macrophages, and vascular growth was restored by AST-120 treatment following the hind-limb ischemia in diabetic mice. Analogous findings were found when the data were analyzed according to the ratio of $\mathrm{iNOS}^{+}$cells and the total number of cells in the ischemic limb.

\section{Pro-inflammatory, Anti-inflammatory, and Angiogenic Cytokine Expression in the Ischemic Limbs of DM + AST- 120 Mice}

Two weeks after the hind-limb ischemia surgery, the levels of TNF-a, IL-6, Cxcl2, and MCP-1 protein were significantly increased in the ischemic tissues of DM mice when compared with those in the WT mice (Fig. 5). In the AST-120 treated DM mice, the up-regulated levels of TNF-a, IL-6, and MCP-1 protein were reversed, and the level of Cxcl 2 was partially reversed. The levels of IL-10 and VEGF were significantly decreased in the DM groups; however, AST-120 treatment did not revere these effects.

\section{Effect of AGEs on Macrophage Polarization in BMDMs:}

The binding assay demonstrated that AST-120 could adsorb and decrease the content of AGEs in the culture media (Fig. 6A, 6B). Macrophages treated with AGEs had significant increase in iNOS mRNA transcription following incubation for 48 hours. In contrast, AST-120 pre-treated AGEs group had a significant decrease in iNOS mRNA transcription when compared with the AGE-alone group (Fig. 6C). In case of M2 markers, AGEs significantly downregulated Arg1 mRNA transcription. AST-120 pretreated AGEs reversed the decrease in Arg1 mRNA when compared with the AGEs-alone group (Fig. 6D). To mimic a diabetic condition, these experiments were repeated after incubation in high-glucose medium for 
48 hours (Fig. 6E). High-glucose incubation resulted in an increase in iNOS mRNA transcription when compared with that in the control medium. The effects of AGEs and pre-treated AGEs in the high-glucose condition were similar to those in the normal-glucose condition: AGE-treated BMDMs had higher iNOS mRNA transcription when compared with macrophages treated with glucose alone. Pre-treatment with AST-120 partially reversed the change in iNOS mRNA transcription induced by AGEs.

\section{Discussion}

The main findings of this study can be summarized as follows: (1) oral AST-120 reduced the plasma levels of AGEs, probably, via absorption of dietary AGEs; (2) AST-120 therapy decreased circulating and tissue levels of AGEs and resulted in improvements in neovascularization and blood flow recovery in a hind-limb ischemia model in diabetic mice; and (3) the polarity of macrophages in the ischemic tissues was predominantly pro-inflammatory M1 phenotype in diabetic mice, and AST-120 therapy reversed this polarity into the pro-angiogenic M2 phenotype.

In patients with diabetes, neovascularization is insufficient to overcome the loss of blood flow that occurs due to arterial narrowing or occlusion [2]. Angiogenesis-defined as the sprouting of new blood vessels from pre-existing vascular structures-is a physiological reaction to tissue ischemia [18]. This process starts with the degradation of non-fibrillar collagens in the basement membrane, followed by migration and proliferation of pre-existing vascular endothelial cells or circulating endothelial progenitor cells (EPCs) [19]. Angiogenesis is initiated by hypoxia and inflammation, and it involves angiogenic, antiangiogenic, and maturation factors [20]. Previous studies have found that alterations in VEGF expression, attenuation of monocyte migratory ability, and impaired EPC mobilization contribute to impairments in neovascularization in diabetes [21, 22]. Formation of AGEs is one of the major mechanisms responsible for vascular damage in diabetes [23]. In RAGE knockout mice, collateral growth and blood flow recovery are reduced when compared with WT mice [24]. Blockade of the formation of AGEs by aminoguanidine also helps in the restoration of perfusion in ischemic tissues [25]. Our data are, broadly, in line with these findings; however, they also provide novel insights into the pathological mechanisms and possible therapeutic approaches.

Many agents have been developed to attenuate the damage induced by AGEs, including inhibitors and breakers of AGEs, antioxidants, natural substances, and anti-inflammatory molecules [23]. Diet-derived AGEs are essential sources of AGEs, and the removal of exogenous AGEs is a potential approach to reduce serum levels of AGEs. It has been demonstrated in healthy subjects that dietary restriction alone could reduce serum levels of AGEs by $30-40 \%[26,27]$. In patients with diabetes or renal failure, restriction of dietary AGEs could attenuate tissue injury related to AGEs [28, 29]. AST-120 is an oral adsorbent that can bind to many low-molecular-weight (100-10,000 kDa) compounds. It has been used to reduce a variety of uremic toxins in patients with CKD, with concomitant improvements in the intimalmedial thickness and flow-mediated dilatation [30, 31]. AST-120 could completely adsorb CML, a wellrecognized food-derived AGE, and reduce serum levels of AGEs in patients with CKD [12]. For the first time, we demonstrated that AST-120 could also reduce serum levels of AGEs in diabetic mice with normal 
renal functions. Circulating AGEs were reduced by approximately $67 \%$ in diabetic mice treated with AST120 , resulting in a serum level equal to that in non-diabetic mice. Although the adsorptive property of AST120 was not examined in gastric or intestinal fluids, in vitro data suggest that AST-120 could adsorb AGEs in food and, subsequently, decrease the serum levels of AGEs. The evidence collectively suggests that adsorption of exogenous AGEs may be a potential therapeutic approach to attenuate ischemic injury in diabetes. Further studies are required to clarify the optimal dose of AST-120 and the degree of reduction in the levels of AGEs required to produce clinical benefits.

Few studies have addressed the underlying mechanisms via which AGEs attenuate neovascularization. Tamarat et al. demonstrated that the formation of AGEs reduced degradation of the extracellular matrix degradation and, subsequently, abrogated the angiogenic process in diabetic mice [25]. Tanii et al. demonstrated that AGEs disturb the recruitment and functions of pericytes via the platelet-derived growth factor-BB/protein kinase $\mathrm{C}$ axis, which regulates the maturation of capillary vessels [32]. Our findings demonstrated that the deleterious effects of AGEs on neovascularization might be related to inflammatory activation in ischemic tissues through the binding of AGEs with RAGE. Macrophages are the principal cells that participate in the inflammatory and angiogenic processes following tissue ischemia. We demonstrated a significant increase in the macrophage infiltration in ischemic tissues in diabetic mice, which was reversed by reducing the AGEs with AST-120 therapy. The infiltration by macrophages was accompanied by increases in pro-inflammatory cytokines and decreases in proangiogenic cytokines. The effects of AGEs on macrophages may be mediated through the binding to RAGE, as demonstrated by the co-expression of RAGE and F4/80 positive cells in ischemic tissues. AST120 decreased not only the circulating AGEs but also the AGE-positive cells, RAGE-positive cells, and RAGE as well as F4/80 double-positive cells in ischemic tissues. The detailed mechanism of enhanced activation of macrophages by AGEs, mainly via the RAGE/NF-KB pathway was demonstrated by Jin et al. [33].

In addition to inflammatory activation, macrophages respond to environmental signals and transform into different functional phenotypes, ranging from pro-inflammatory (classic M1 activation) to proangiogenic phenotypes (alternative M2 activation) [34,35]. The pro-angiogenic property is not demonstrated in all subsets of macrophages. M2 macrophages promote angiogenesis by producing proangiogenic cytokines and growth factors. In diabetic mice, we found the pro-inflammatory M1 phenotype to be the predominant one in ischemic tissues. In non-diabetic mice, the predominant macrophages were of the pro-angiogenic M2 phenotype. A comparable change in pro-inflammatory cytokines (TNF-a, IL-6, MCP-1, and Cxc16) and pro-angiogenic cytokine (VEGF) expression supports the role of macrophage polarization in the angiogenic process. AST-120 reversed the M1/M2 polarization, resulting in subsequent improvements in neovascularization. The effects of AGEs on macrophage polarity were demonstrated in in vitro studies as well. Either AGEs or high-glucose conditions enhance the transformation of macrophages into M1 phenotype. Administration of AST-120 could reverse the change in polarity.

The impact of macrophage polarization on the angiogenic process was investigated in previous studies. Angiogenic growth factors and cytokines are highly expressed in M2 rather than M1 macrophages, and 
M2 macrophages promote tube formation by these secretory factors in vitro. [36, 37]. Neutralizing these growth factors could impair the M2-induced angiogenesis [37]. In animal models, the infusion of antiinflammatory M2 macrophages but not pro-inflammatory M1 macrophages promotes angiogenesis [37]. Collectively, AGE-RAGE related alternations in macrophage polarization might be a probable mechanism underlying the microangiopathy in diabetes.

Some limitations of this study must be acknowledged. First, AGEs are recognized as some of the most potent toxins in diabetes that can be reduced by AST-120. Although mice without CKD were used in this experiment, we could not exclude the possibility that the benefits were derived from reductions in other toxins. Second, the causal relationship between AGEs and neovascularization and detailed mechanical pathways involved could not be proven directly in this animal model.

\section{Conclusions}

AGEs may have deleterious effects on neovascularization by altering the polarization of macrophages from the angiogenic to the pro-inflammatory phenotype in diabetes. Administration of AST-120 to diabetic mice improved neovascularization and blood flow recovery in ischemic tissues, probably, by decreasing the intestinal absorption of AGEs. AST-120 may be an effective novel therapeutic agent to facilitate neovascularization and improve the outcomes in patients with diabetic and peripheral arterial disease.

\section{Abbreviations}

AGEs advanced glycation end products

RAGE receptor for advanced glycation end products

CML carboxymethyl lysine

CKD chronic kidney disease

ELISA enzyme-linked immunosorbent assay

STZ streptozotocin

IPGPTTs intraperitoneal glucose tolerance tests

TNF tumor necrosis factor

IL interleukin

Cxcl2 C-X-C motif ligand 2

MCP monocyte chemoattractant protein 
M-CSF plasma macrophage colony-stimulating factor

VEGF vascular endothelial growth factor

iNOS inducible nitric oxide synthase

BMDMs bone marrow-derived macrophages

PCR polymerase chain reaction

EPCs endothelial progenitor cells

\section{Declarations}

All authors declare that they have no conflict of interest.

Availability of data and materials: Not applicable.

ETHICS APPROVAL: All experimental procedures and protocols involving animals were in accordance with the standards of the Institutional Animal Care Committee of National Yang-Ming University (Taipei, Taiwan), and complied with the Guide for the Care and Use of Laboratory Animals.

CONSENT FOR PUBLICATION: Not applicable.

COMPETING INTERESTS: The authors declare that they have no competing interests.

FUNDING:

This study was supported in part by research grants from the Novel Bioengineering and Technological Approaches to Solve Two Major Health Problems in Taiwan program, sponsored by the Taiwan Ministry of Science and Technology Academic Excellence Program (MOST-106-2633-B-009-003); Taipei Veterans General Hospital (VGH-V100E2-002 and VGHUST103-G7-2-1); the National Taiwan University Hospital, Hsinchu Branch (107-HCHOO2 and 108-HCH004); and the Ministry of Science and Technology (MOST105-2314-B-002-119, 106-2314-B-002-173-MY3, MOHW 106-TDU-B-211-113001). The funding institutions took no part in the study design, data collection or analysis, publication intent, or manuscript preparation.

\section{AUTHORS' CONTRIBUTIONS:}

Hsin -Lei Huang, Ting-Yung Chang and Po-Hsun Huang conceived of the presented idea. Hsin -Lei Huang developed the theory and performed the experiments, and analytical methods. I-Chun Chen, Fu-Chen Yang, Nien-Jung Chen help to build the method of in vitro study. Ting-Yung Chang, Ruey-Hsing Chou, DerCherng Tarng, Shing-Jong Lin help to build the method of in vivo study. Hsin -Lei Huang, Chin-Sung Kuo, Chih-Cheng Wu and Po-Hsun Huang took the lead in writing the manuscript. Po-Hsun Huang encouraged the investigate and supervised the findings of this work. All authors discussed the results and contributed to the final manuscript. 
Nil.

\section{References}

1. Meigs JB, Singer DE, Sullivan LM, Dukes KA, D'Agostino RB, Nathan DM, Wagner EH, Kaplan SH, Greenfield S. Metabolic control and prevalent cardiovascular disease in non-insulin-dependent diabetes mellitus (NIDDM): The NIDDM Patient Outcome Research Team. Am J Med. 1997;102(1):38-47.

2. Abaci A, Oguzhan A, Kahraman S, Eryol NK, Unal S, Arinc H, Ergin A. Effect of diabetes mellitus on formation of coronary collateral vessels. Circulation. 1999;99(17):2239-42.

3. Bierhaus A, Haslbeck KM, Humpert PM, Liliensiek B, Dehmer T, Morcos M, Sayed AA, Andrassy M, Schiekofer S, Schneider JG, et al. Loss of pain perception in diabetes is dependent on a receptor of the immunoglobulin superfamily. J Clin Investig. 2004;114(12):1741-51.

4. Toth C, Rong LL, Yang C, Martinez J, Song F, Ramji N, Brussee V, Liu W, Durand J, Nguyen MD, et al. Receptor for advanced glycation end products (RAGEs) and experimental diabetic neuropathy. Diabetes. 2008;57(4):1002-17.

5. Jiang J, Chen P, Chen J, Yu X, Xie D, Mei C, Xiong F, Shi W, Zhou W, Liu X, et al. Accumulation of tissue advanced glycation end products correlated with glucose exposure dose and associated with cardiovascular morbidity in patients on peritoneal dialysis. Atherosclerosis. 2012;224(1):187-94.

6. Yan SF, Ramasamy R, Schmidt AM. Mechanisms of disease: advanced glycation end-products and their receptor in inflammation and diabetes complications. Nature clinical practice Endocrinology metabolism. 2008;4(5):285-93.

7. Barlovic DP, Soro-Paavonen A, Jandeleit-Dahm KA. RAGE biology, atherosclerosis and diabetes. Clinical science. 2011;121(2):43-55.

8. Yan SF, Ramasamy R, Schmidt AM. Receptor for AGE (RAGE) and its ligands-cast into leading roles in diabetes and the inflammatory response. Journal of molecular medicine. 2009;87(3):235-47.

9. Basta G, Lazzerini G, Massaro M, Simoncini T, Tanganelli P, Fu C, Kislinger T, Stern DM, Schmidt AM, De Caterina R. Advanced glycation end products activate endothelium through signal-transduction receptor RAGE: a mechanism for amplification of inflammatory responses. Circulation. 2002;105(7):816-22.

10. Ritthaler U, Deng Y, Zhang Y, Greten J, Abel M, Sido B, Allenberg J, Otto G, Roth H, Bierhaus A, et al. Expression of receptors for advanced glycation end products in peripheral occlusive vascular disease. Am J Pathol. 1995;146(3):688-94.

11. Soulis T, Thallas V, Youssef S, Gilbert RE, McWilliam BG, Murray-McIntosh RP, Cooper ME. Advanced glycation end products and their receptors co-localise in rat organs susceptible to diabetic microvascular injury. Diabetologia. 1997;40(6):619-28. 
12. Ueda S, Yamagishi S, Takeuchi M, Kohno K, Shibata R, Matsumoto Y, Kaneyuki U, Fujimura T, Hayashida A, Okuda S. Oral adsorbent AST-120 decreases serum levels of AGEs in patients with chronic renal failure. Molecular medicine. 2006;12(7-8):180-4.

13. Kislinger T, Fu C, Huber B, Qu W, Taguchi A, Du Yan S, Hofmann M, Yan SF, Pischetsrieder M, Stern D, et al. N(epsilon)-(carboxymethyl)lysine adducts of proteins are ligands for receptor for advanced glycation end products that activate cell signaling pathways and modulate gene expression. J Biol Chem. 1999;274(44):31740-9.

14. Yamagishi S, Nakamura K, Matsui T, Inoue H, Takeuchi M. Oral administration of AST-120 (Kremezin) is a promising therapeutic strategy for advanced glycation end product (AGE)-related disorders. Medical hypotheses. 2007;69(3):666-8.

15. Huang PH, Lin CP, Wang $\mathrm{CH}$, Chiang $\mathrm{CH}$, Tsai HY, Chen JS, Lin FY, Leu HB, Wu TC, Chen JW, et al. Niacin improves ischemia-induced neovascularization in diabetic mice by enhancement of endothelial progenitor cell functions independent of changes in plasma lipids. Angiogenesis. 2012;15(3):377-89.

16. Lawrence T, Natoli G. Transcriptional regulation of macrophage polarization: enabling diversity with identity. Nature reviews Immunology. 2011;11(11):750-61.

17. Geissmann F, Gordon S, Hume DA, Mowat AM, Randolph GJ. Unravelling mononuclear phagocyte heterogeneity. Nature reviews Immunology. 2010;10(6):453-60.

18. Folkman J. Seminars in Medicine of the Beth Israel Hospital, Boston. Clinical applications of research on angiogenesis. N Engl J Med. 1995;333(26):1757-63.

19. Folkman J. Angiogenesis in cancer, vascular, rheumatoid and other disease. Nat Med. 1995;1(1):2731.

20. Takahashi T, Kalka C, Masuda H, Chen D, Silver M, Kearney M, Magner M, Isner JM, Asahara T. Ischemia- and cytokine-induced mobilization of bone marrow-derived endothelial progenitor cells for neovascularization. Nat Med. 1999;5(4):434-8.

21. Rivard A, Silver M, Chen D, Kearney M, Magner M, Annex B, Peters K, Isner JM. Rescue of diabetesrelated impairment of angiogenesis by intramuscular gene therapy with adeno-VEGF. Am J Pathol. 1999;154(2):355-63.

22. Waltenberger J, Lange J, Kranz A. Vascular endothelial growth factor-A-induced chemotaxis of monocytes is attenuated in patients with diabetes mellitus: A potential predictor for the individual capacity to develop collaterals. Circulation. 2000;102(2):185-90.

23. Goldin A, Beckman JA, Schmidt AM, Creager MA. Advanced glycation end products: sparking the development of diabetic vascular injury. Circulation. 2006;114(6):597-605.

24. Hansen LM, Gupta D, Joseph G, Weiss D, Taylor WR. The receptor for advanced glycation end products impairs collateral formation in both diabetic and non-diabetic mice. Lab Invest. 2017;97(1):34-42.

25. Tamarat R, Silvestre JS, Huijberts M, Benessiano J, Ebrahimian TG, Duriez M, Wautier MP, Wautier JL, Levy Bl. Blockade of advanced glycation end-product formation restores ischemia-induced 
angiogenesis in diabetic mice. Proc Natl Acad Sci U S A. 2003;100(14):8555-60.

26. Koschinsky T, He CJ, Mitsuhashi T, Bucala R, Liu C, Buenting C, Heitmann K, Vlassara H. Orally absorbed reactive glycation products (glycotoxins): an environmental risk factor in diabetic nephropathy. Proc Natl Acad Sci USA. 1997;94(12):6474-9.

27. Goldberg T, Cai W, Peppa M, Dardaine V, Baliga BS, Uribarri J, Vlassara H. Advanced glycoxidation end products in commonly consumed foods. J Am Diet Assoc. 2004;104(8):1287-91.

28. Uribarri J, Peppa M, Cai W, Goldberg T, Lu M, He C, Vlassara H. Restriction of dietary glycotoxins reduces excessive advanced glycation end products in renal failure patients. J Am Soc Nephrol. 2003;14(3):728-31.

29. Vlassara H, Cai W, Crandall J, Goldberg T, Oberstein R, Dardaine V, Peppa M, Rayfield EJ. Inflammatory mediators are induced by dietary glycotoxins, a major risk factor for diabetic angiopathy. Proc Natl Acad Sci USA. 2002;99(24):15596-601.

30. Nakamura T, Kawagoe Y, Matsuda T, Ueda Y, Shimada N, Ebihara I, Koide H. Oral ADSORBENT AST120 decreases carotid intima-media thickness and arterial stiffness in patients with chronic renal failure. Kidney Blood Press Res. 2004;27(2):121-6.

31. Yu M, Kim YJ, Kang DH. Indoxyl sulfate-induced endothelial dysfunction in patients with chronic kidney disease via an induction of oxidative stress. Clin J Am Soc Nephrol. 2011;6(1):30-9.

32. Tanii M, Yonemitsu Y, Fujii T, Shikada Y, Kohno R, Onimaru M, Okano S, Inoue M, Hasegawa M, Onohara $\mathrm{T}$, et al. Diabetic microangiopathy in ischemic limb is a disease of disturbance of the platelet-derived growth factor-BB/protein kinase $\mathrm{C}$ axis but not of impaired expression of angiogenic factors. Circ Res. 2006;98(1):55-62.

33. Jin X, Yao T, Zhou Z, Zhu J, Zhang S, Hu W, Shen C. Advanced Glycation End Products Enhance Macrophages Polarization into M1 Phenotype through Activating RAGE/NF-kappaB Pathway. Biomed Res Int. 2015;2015:732450.

34. Biswas SK, Mantovani A. Macrophage plasticity and interaction with lymphocyte subsets: cancer as a paradigm. Nature immunology. 2010;11(10):889-96.

35. Sica A, Mantovani A. Macrophage plasticity and polarization: in vivo veritas. J Clin Investig. 2012;122(3):787-95.

36. Takeda Y, Costa S, Delamarre E, Roncal C, Leite de Oliveira R, Squadrito ML, Finisguerra V, Deschoemaeker S, Bruyere F, Wenes M, et al. Macrophage skewing by Phd2 haplodeficiency prevents ischaemia by inducing arteriogenesis. Nature. 2011;479(7371):122-6.

37. Jetten N, Verbruggen S, Gijbels MJ, Post MJ, De Winther MP, Donners MM. Anti-inflammatory M2, but not pro-inflammatory M1 macrophages promote angiogenesis in vivo. Angiogenesis. 2014;17(1):109-18.

\section{Figures}



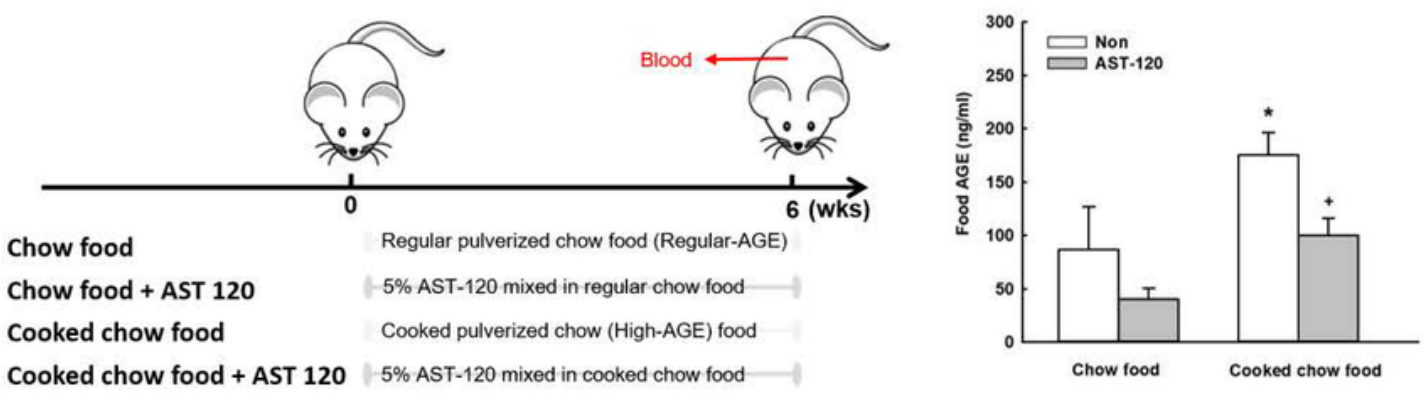

C

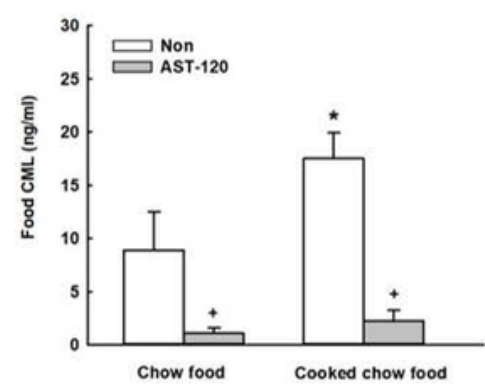

D

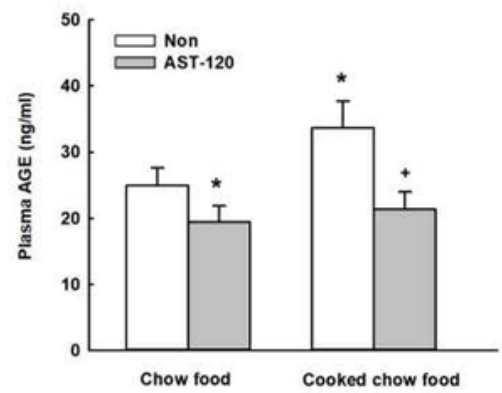

E

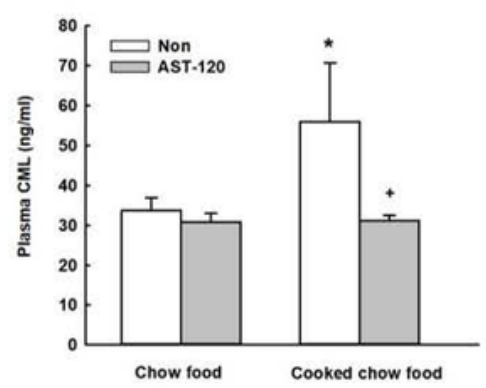

F

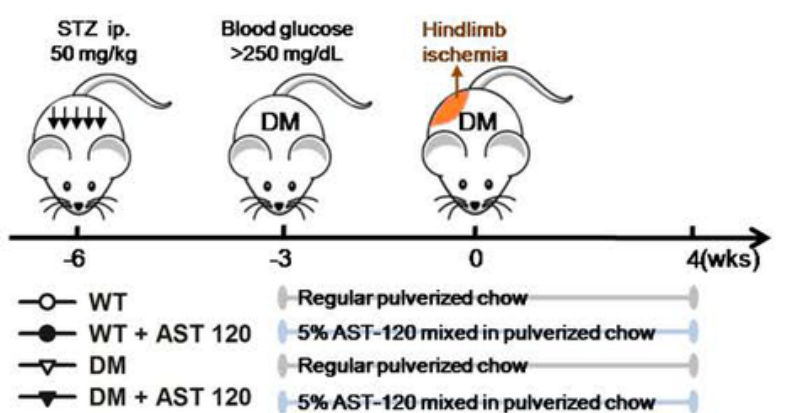

G
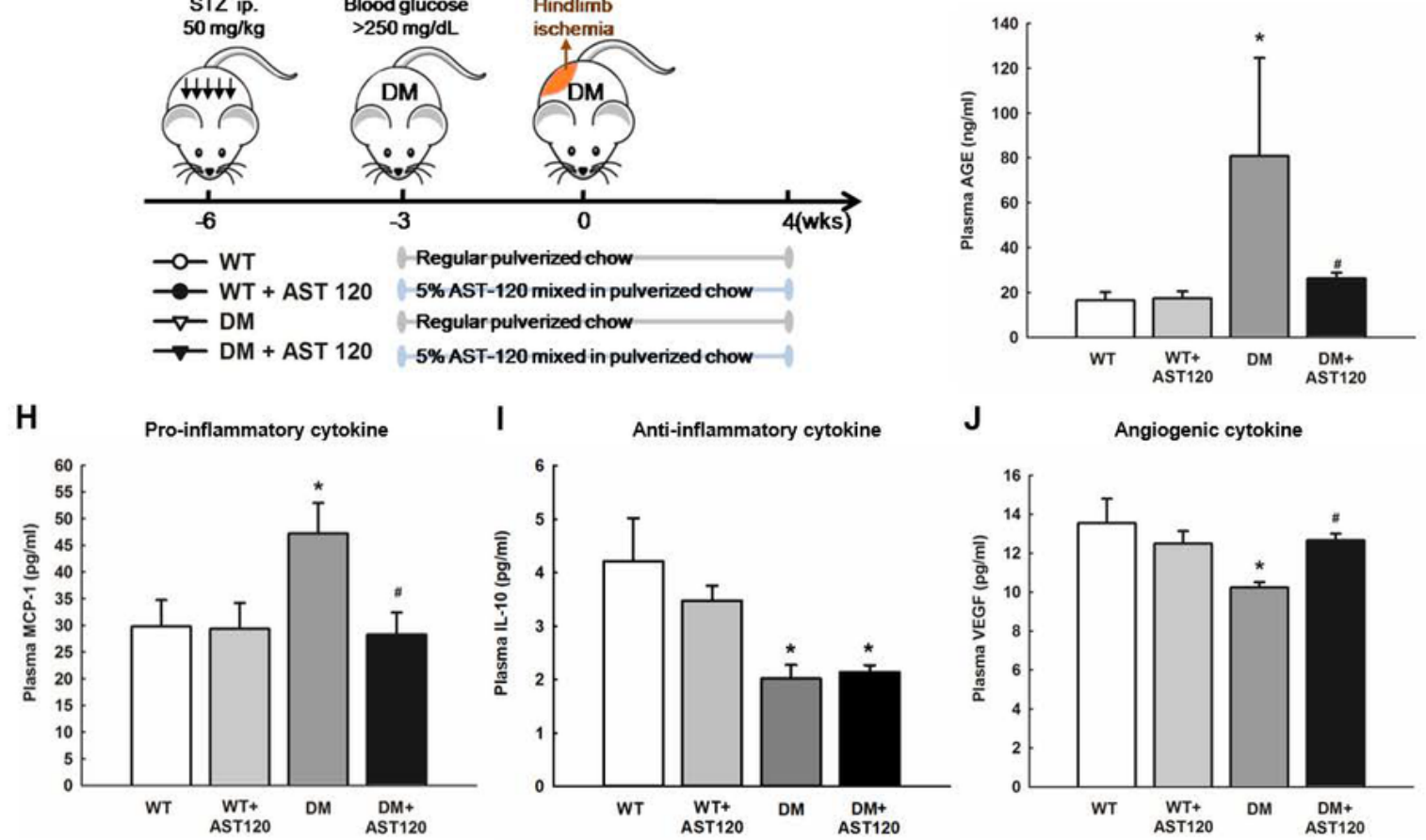

\section{Figure 1}

AST-120 reduce dietary advanced glycation end products (AGEs) absorption in diabetic mice. (A) Mice were divided into four groups: mice treated with regular pulverized chow food (Chow food), mice treated with 5\% AST-120 mixed into regular chow food (Chow food + AST-120), mice treated with cooked pulverized chow food (Cooked chow food), and mice treated with 5\% AST-120 mixed into cooked chow food (Cooked chow food + AST-120). To invested the binding effect of AST-120, the supernatant from 
four group's food was measured by AGE (B) and CML (C) ELISA kits. After 6 weeks treatment, mice plasma AGE (D) and CML (E) levels were measured. (F) To study the AST-120 treatment in diabetic mice, mice were divided into four groups: WT, WT + AST-120, DM, and DM + AST-120. Plasma AGE (G), MCP-1 $(\mathrm{H}), \mathrm{IL}-10(\mathrm{I})$, and VEGF $(\mathrm{J})$ levels were measured 14 days after hindlimb ischemia surgery. ${ }^{*}<0.05 \mathrm{vs}$. WT, **P $<0.01$ vs. WT, \#P $<0.05$ vs. $D M ; n=11$ per group.

A

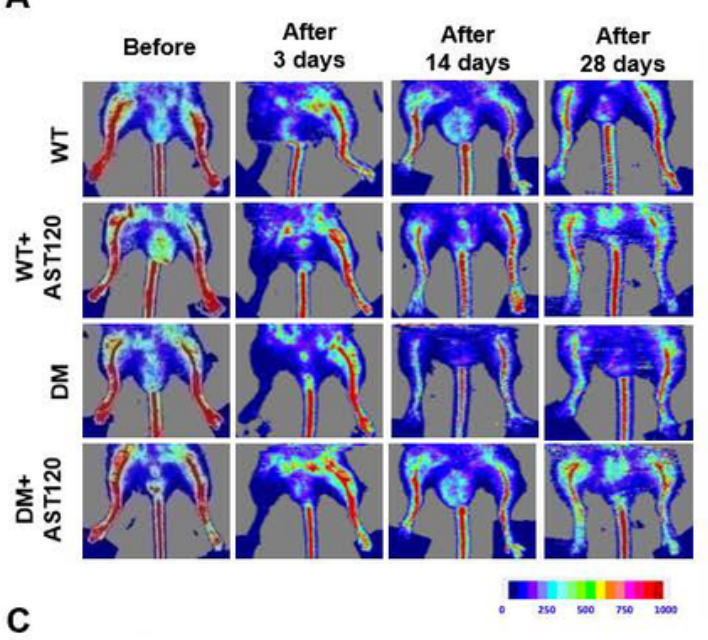

C

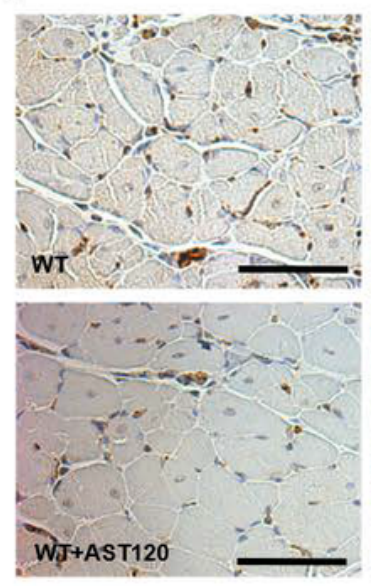

E

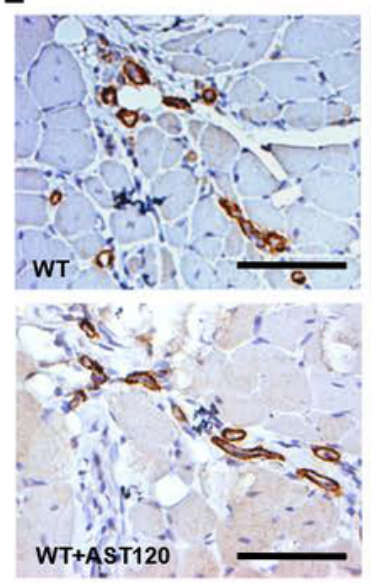

B
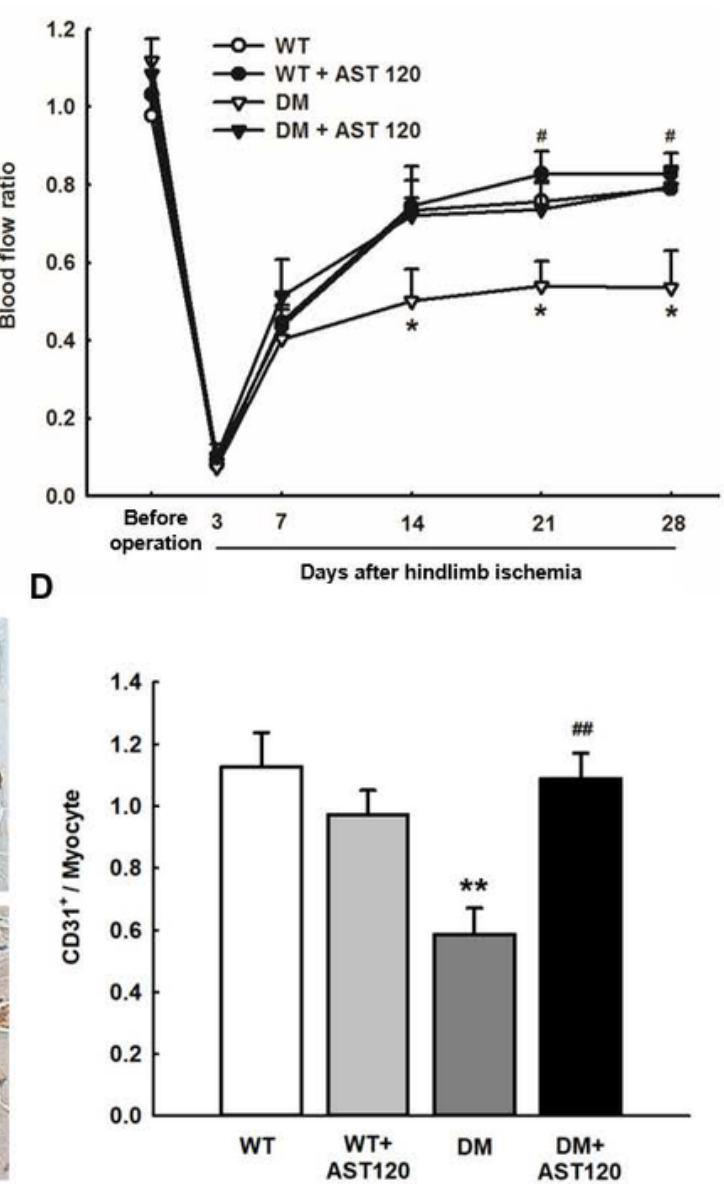

F
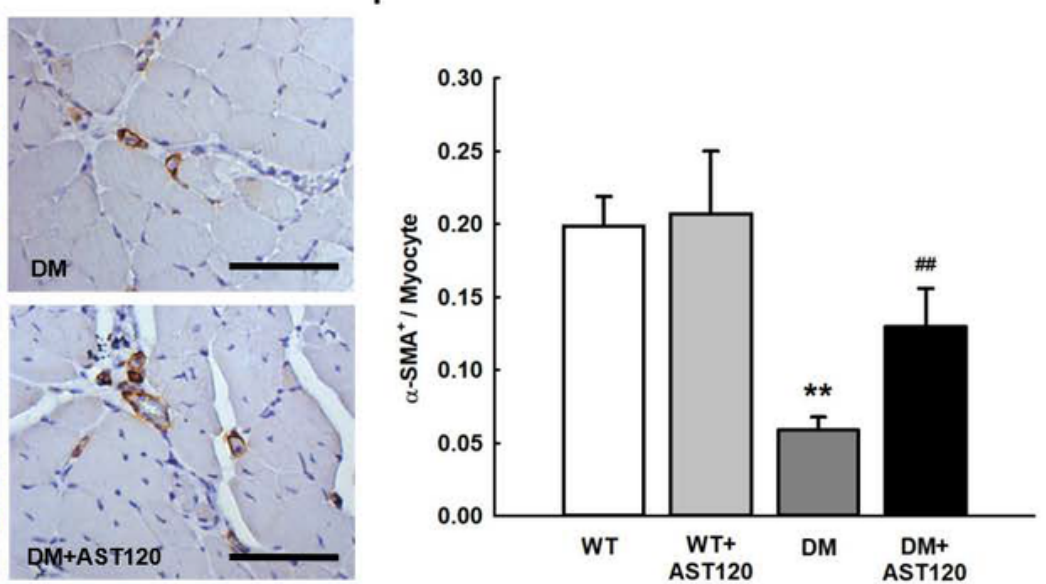

Figure 2 
AST-120 treatment improved blood reperfusion after hindlimb ischemia surgery in diabetic mice. (A) Blood reperfusion in ischemic limbs, measured preoperatively and $3,7,14,21$, and 28 days after hindlimb ischemia surgery by laser Doppler $(n=9)$. (B) Quantification of blood perfusion in mouse limbs. On day 14 after injury, tissue samples were collected and the following immunostaining procedures were performed. (C) Immunochemical staining of ischemic muscle cross sections for CD31 (brown) and hematoxylin (blue) in the indicated mouse groups. (D) Quantification of CD31+ capillary density in the ischemic muscle sections. (E) Immunochemical staining of ischemic muscle cross sections for a-SMA (brown) and hematoxylin (blue) in the indicated mouse groups. (F) Quantification of a-SMA+ small artery density in the ischemic muscle sections. ${ }^{*} \mathrm{P}<0.05$ vs. WT, ${ }^{\star *} \mathrm{P}<0.01 \mathrm{vs}$. WT, \#P<0.05 vs. DM; $n=9$ per group. \#\#P $<0.01$ vs. DM; $\mathrm{n}=7$ per group. 
A

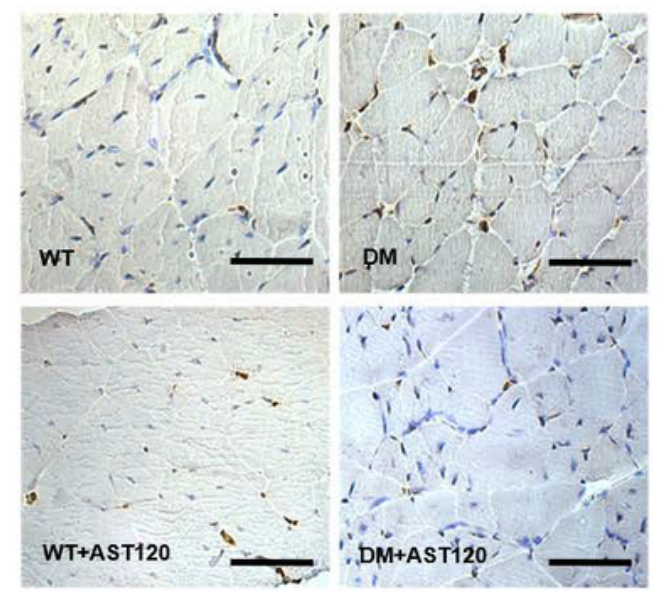

C
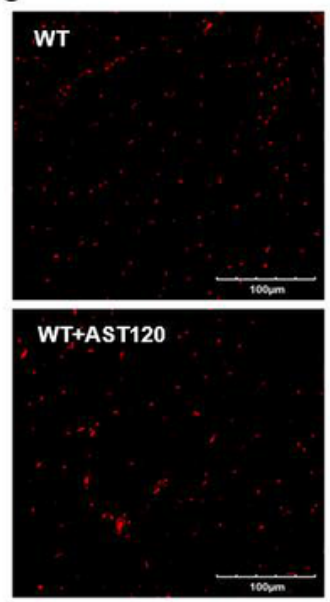

E
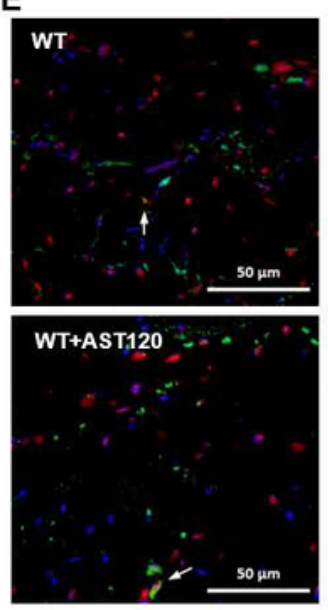

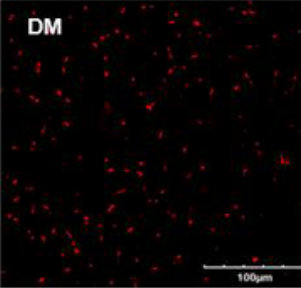

DM+AST120

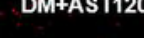

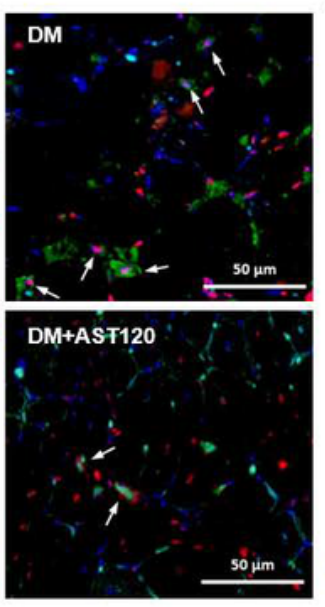

B

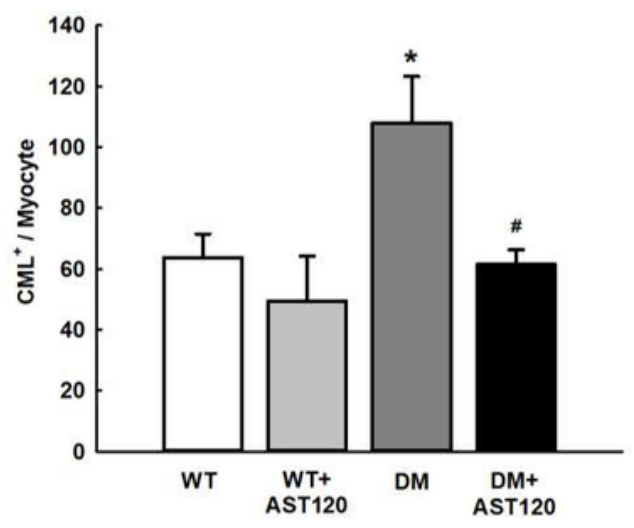

D

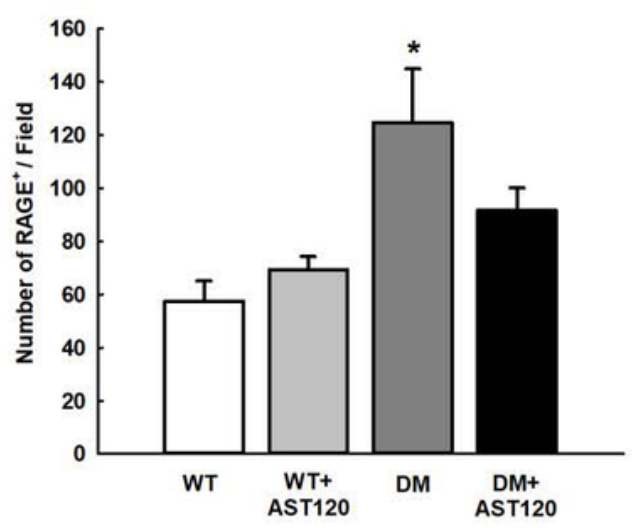

F

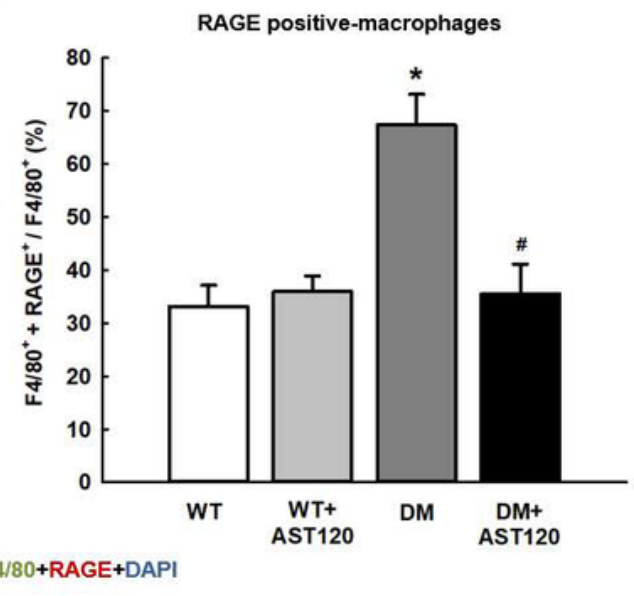

\section{Figure 3}

Expression of CML, RAGE and macrophage infiltration in the ischemic muscles of diabetic mice. On day 14 after injury, the following immunostaining procedures were performed. (A) Immunochemical staining of ischemic muscle cross sections for CML (brown) and hematoxylin (blue) in the indicated mouse groups. (B) Quantification of CML density in the ischemic muscle sections. (C) Immunofluorescence staining of ischemic muscle cross sections for RAGE (red) in the indicated mouse groups. (D) 
Quantification of RAGE density in the ischemic muscle sections. (E) Representative images of immunofluorescence staining of ischemic muscle cross sections for F4/80 (green), RAGE (red), and DAPI (nuclei; blue) in the indicated mouse groups. (F) Quantification of RAGE+ macrophage density in the ischemic muscle sections. ${ }^{*} P<0.05$ vs. WT, \#P<0.05 vs. $D M ; n=7$ per group. ${ }^{*} P<0.05$ vs. WT; $n=7$ per group.

$\begin{array}{ll}\text { A } & \text { wT }\end{array}$
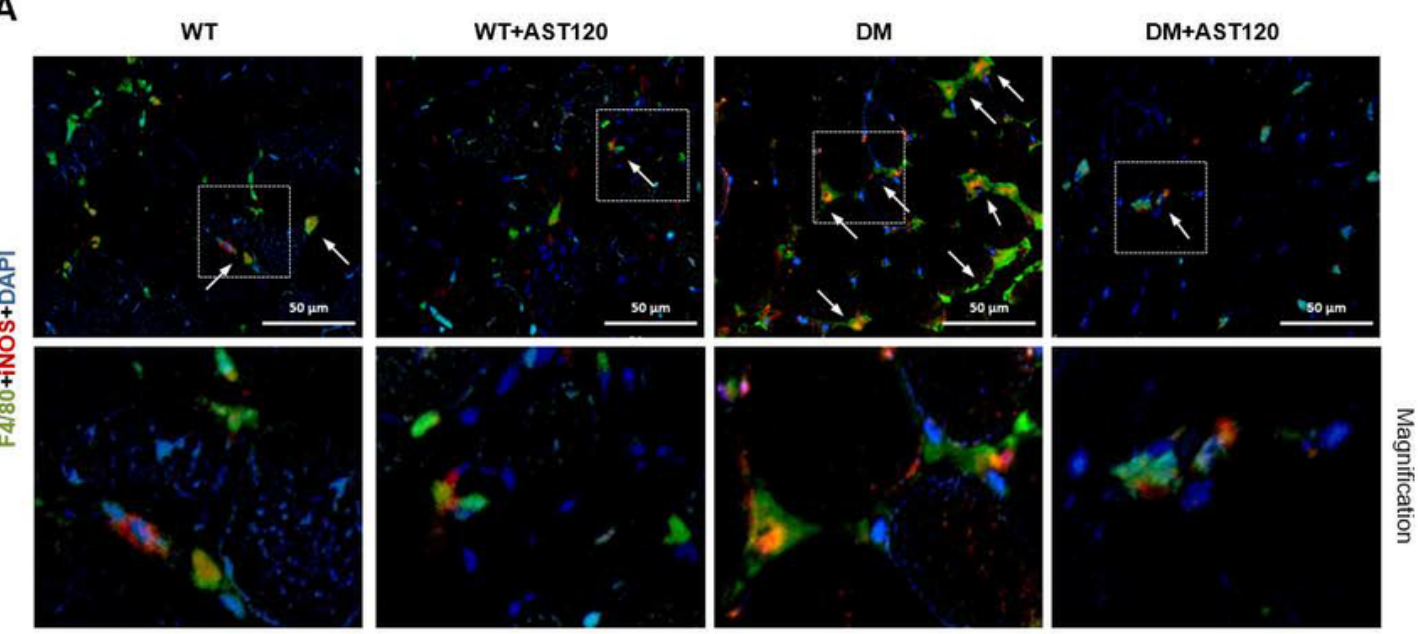

B

WT

WT+AST120

DM

DM+AST120
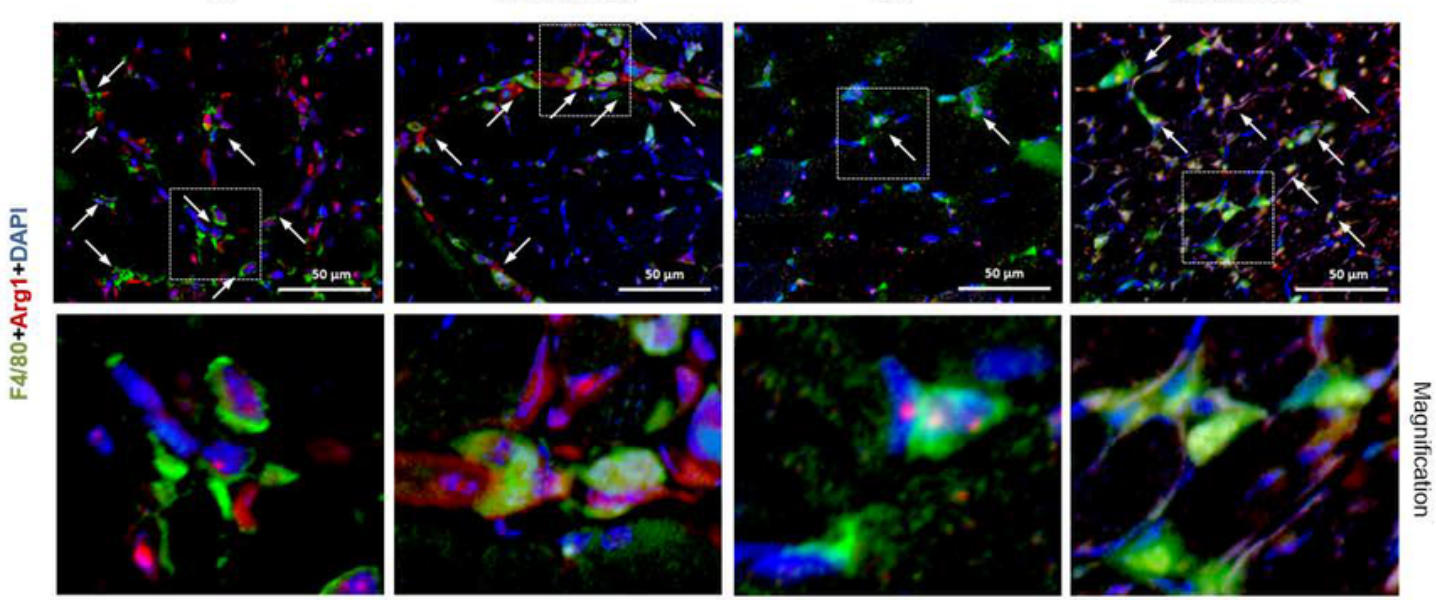

C

D
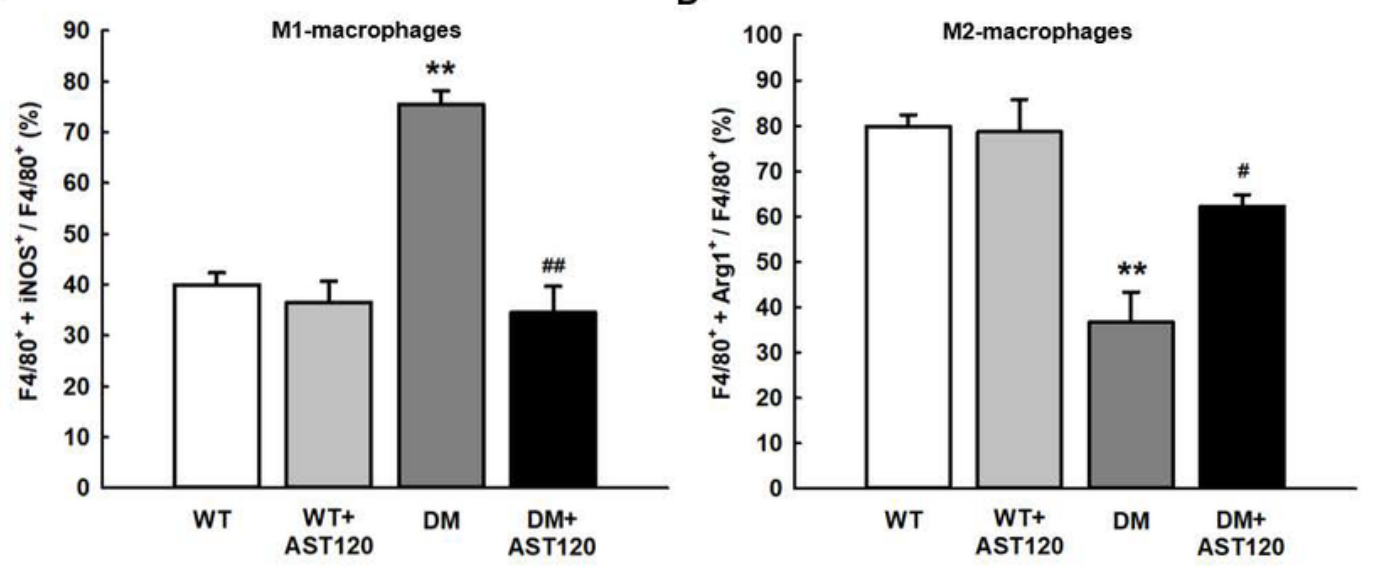

Figure 4 
Effect of AST-120 on macrophage polarization in ischemic limbs of diabetic mice. On day 14 after injury, the following immunofluorescence staining was performed. (A) Invading macrophages were stained for iNOS (M1 marker; red), F4/80 (macrophage marker; green) and DAPI (nuclei; blue) in ischemic muscle cross sections from the indicated mouse groups. (B) Invading macrophages were stained for Arg-1 (M2 marker; red), F4/80 (macrophage marker; green) and DAPI (nuclei; blue) in ischemic muscle cross sections from the indicated mouse groups. Representative images are show in the upper panels and magnified images are shown in the lower panels. (C) Quantification of M1 macrophage infiltration in the ischemic muscle sections. Results are depicted as the percent of total macrophages co-staining for the M1 marker and iNOS. (D) Quantification of M2 macrophage infiltration in the ischemic muscle sections. Results are depicted as the percent of total macrophages co-staining for the $\mathrm{M} 2$ marker and $\operatorname{Arg} 1 .{ }^{*} \mathrm{P}<$ 0.01 vs. WT, \#P< 0.05 vs. DM, \#\#P $<0.01$ vs. DM; $n=7$ per group.

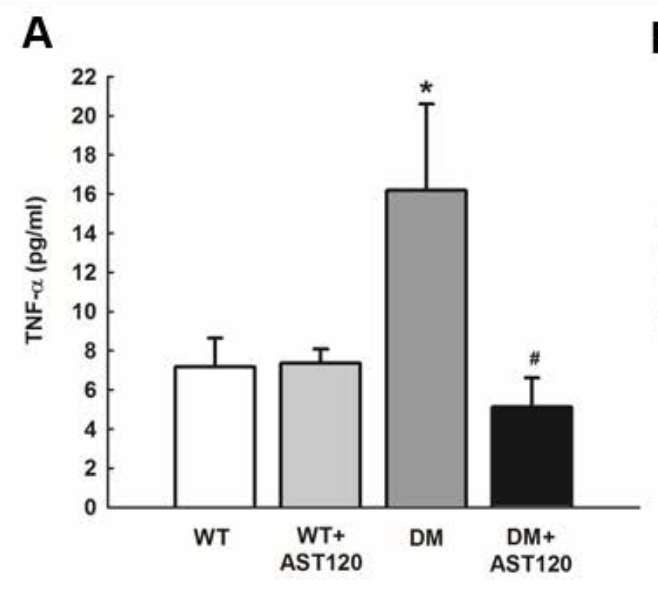

D

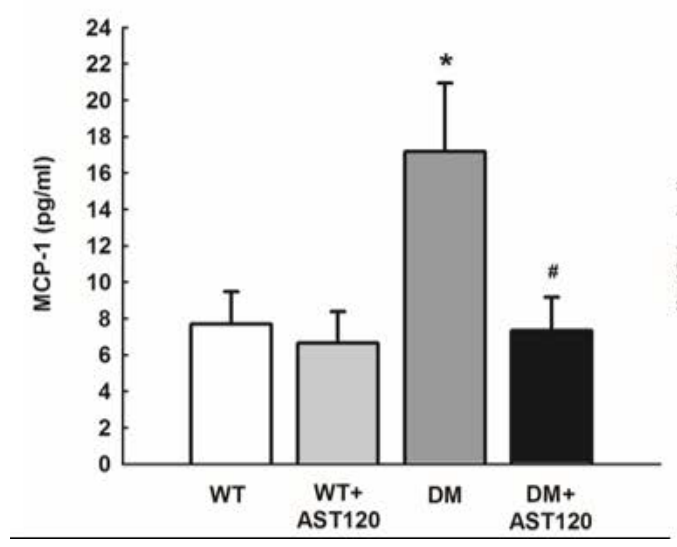

B

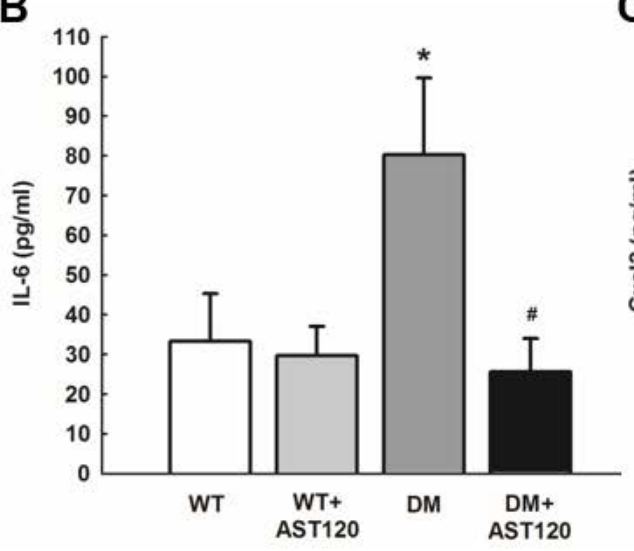

$\mathbf{E}$

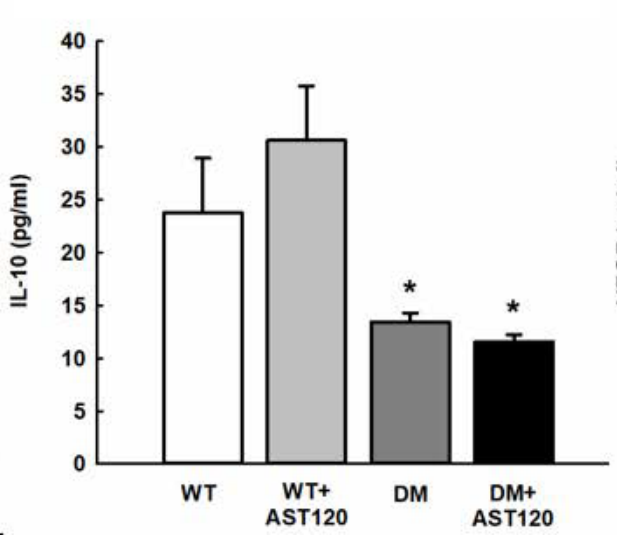

C

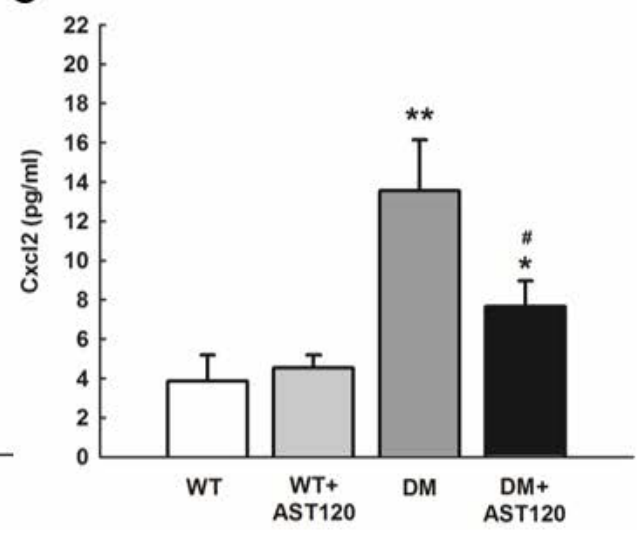

$\mathbf{F}$

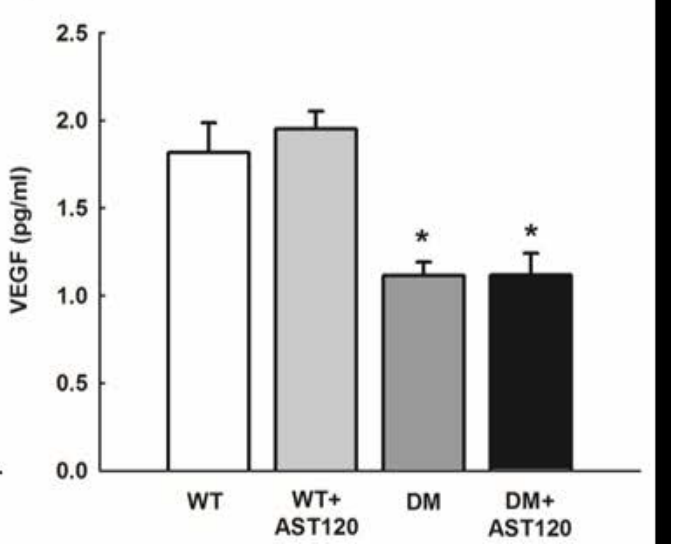

Figure 5

Patterns of macrophage-related cytokine production in ischemic muscles of mice. On day 14 after injury, tissue extract was made from ischemic mouse muscle, and the following protein levels were detected. TNF-a (A), IL-6 (B), Cxcl2 (C), MCP-1 (D), IL-10 (E), and VEGF (F) were measured by multiplex immunoassay. ${ }^{*} P<0.05$ vs. $W T$, \#P $<0.05$ vs. $D M ; n=6$ per group. 
A

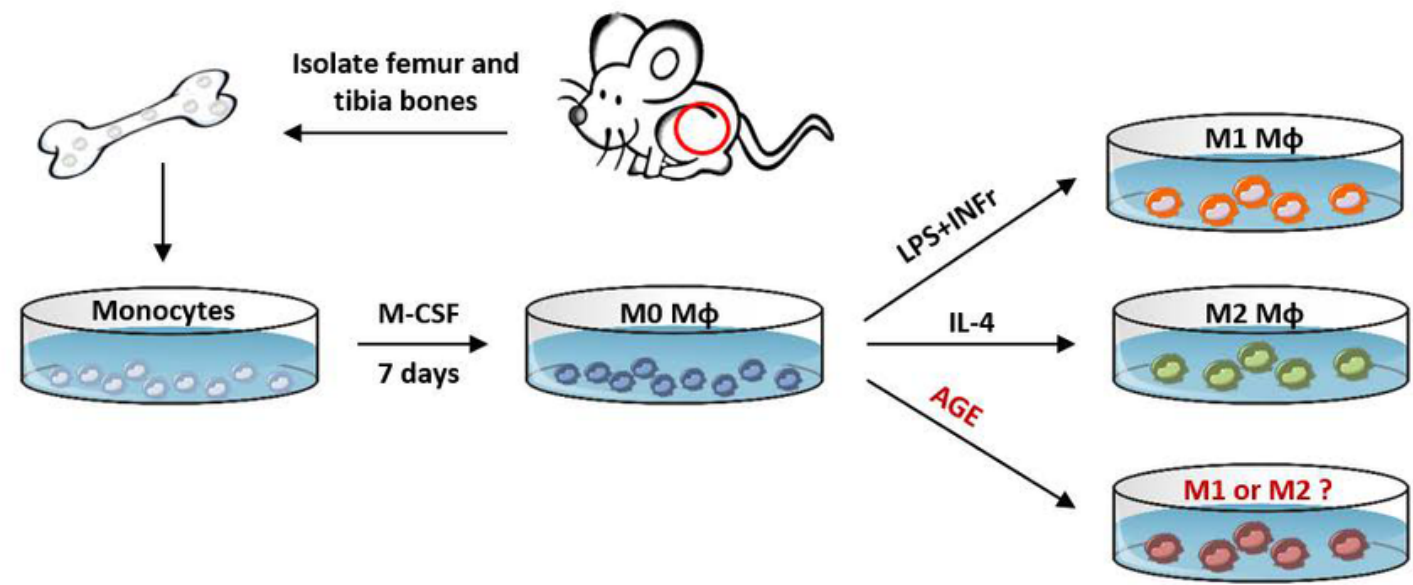

B

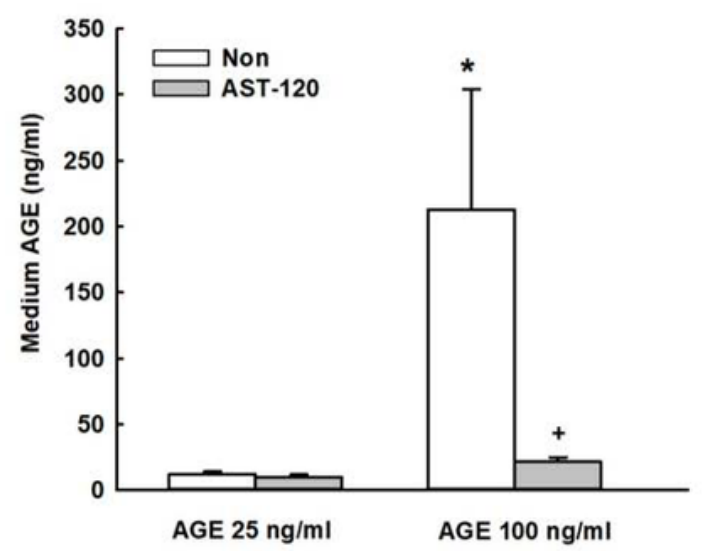

D

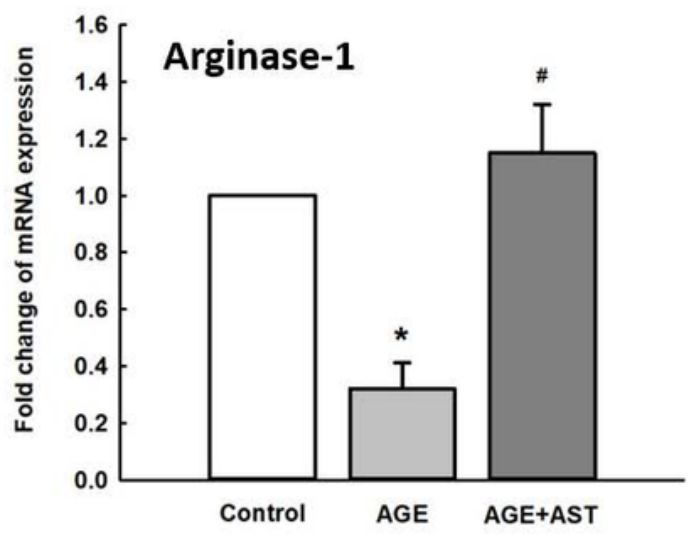

C

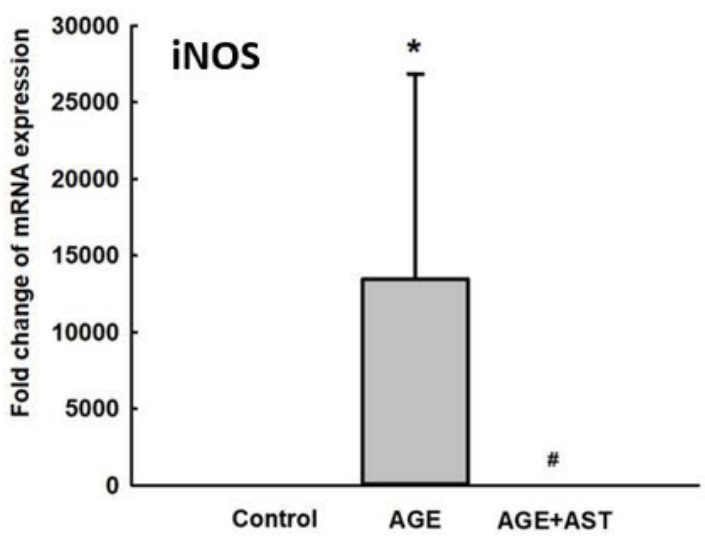

E

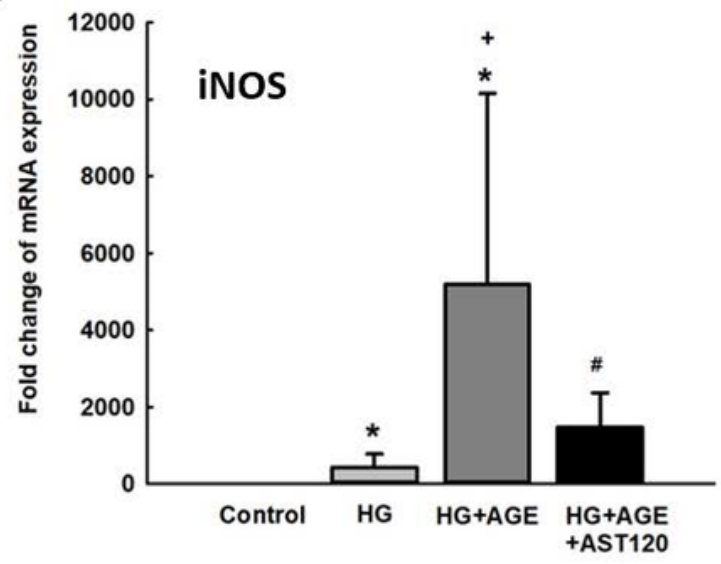

\section{Figure 6}

AGE modulate polarization of bone marrow derived macrophages and the effect of AST-120 treatment. (A) Monocytes were extracted from bone marrow of 6-week wild-type mice and well-differentiated macrophage were harvested at the seventh day. (B) Control, AGE (100 ug/ml), and AST-120-treated AGE medium are used for the binding assay. After 16 hours incubating in $37 \mathrm{oC}$, the supernatant was measured by AGE and CML ELISA kits. Bone marrow-derived macrophages (BMDMs) were incubated for 
48 hours with control, AGE (100 ug/ml), and AST-120-treated AGE medium. (C, D) Macrophages were retrieved and subjected to real-time quantitative PCR to determine the expression of iNOS and Arginase-1. (E) BMDMs were incubated for 48 hours with control, high-glucose condition (25 mmol/L D-glucose; HG), high glucose + AGE (HG+AGE), and high glucose + AST-120-pretreated AGE (HG+AGE+AST-120).

Macrophages were retrieved and subjected to real-time quantitative PCR to determine the expression of iNOS. The expression in control medium were used as the reference. ${ }^{*} \mathrm{P}<0.05$ vs. Control, $+P<0.05$ vs. AGE $100 \mathrm{ng} / \mathrm{ml}, \# \mathrm{P}<0.05 \mathrm{vs}$. AGE treatment; $\mathrm{n}=7$ per group.

\section{Supplementary Files}

This is a list of supplementary files associated with this preprint. Click to download.

- S1.tif

- Supplemental.docx

- S2.tif

- S3.tif

- S4.tif 